\title{
Operating Regime as Rectifier with Power Factor Correction of Two - Quadrant Converter with RNSIC
}

\author{
I.V. Pletea*, D. Alexa, T.C.Goras, R. Chiper, A. Petrichei, C. Nedelcu and A. Alexandrescu \\ Technical University "Gh. Asachi" of Iasi, Faculty of Electronics and Telecommunications, Blv. Carol I, nr, 11, Iasi, \\ cod 700506, Romania
}

\begin{abstract}
A new topology for a two-quadrant converter is presented. In the AC/DC transfer mode the converter works as a rectifier with near sinusoidal input currents (RNSIC), while in the DC/AC transfer mode it works as a square - wave pulse switching inverter. Some suggestions for the converter is characterized by smaller power losses, reduced EMI problems and higher reliability.This new converter topology could have numerous applications. For example, in adjustable speed drives with regenerative braking, wind energy conversion systems and small hydro interconnections with induction generators.
\end{abstract}

\section{INTRODUCTION}

A new converter for two - quadrant is presented in this paper; it is equipped with 6 transistors (e.g. IGBT) having square-wave pulse switching (that is not PWM) operation, as shown in Fig. (1a). When the energy is transferred from the AC side to DC side, the transistors are off and the converter switching) and the converter works as inverter, as show in Fig. (1b).

\section{AC/DC OPERATION MODE}

Which is a module of the two quadrant converter in Fig. (1a). The capacitors $\mathrm{C}_{1}-\mathrm{C}_{6}$ have the same value $\mathrm{C}$ and they

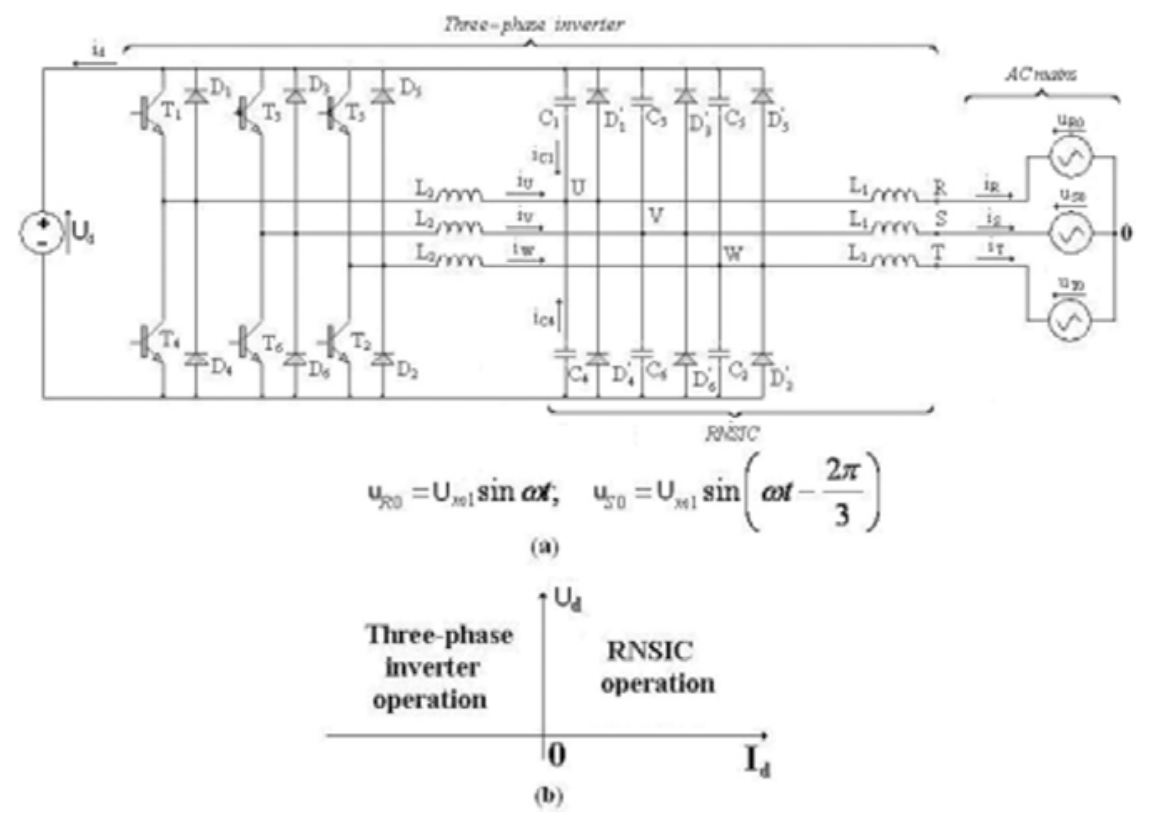

Fig. (1). Converter for two - quadrant with RNSIC; (a) Configuration; (b) Operations

works as a RNSIC (Rectifier with Near Sinusoidal Input Currents), as show in Fig. (2) [7-9]. When the energy is transferred from the DC side to the AC side, the transistors are controlled to conduct for $\theta$ angles (square-wave pulse

*Address correspondence to this author at the Technical University "Gh. Asachi" of Iasi, Faculty of Electronics and Telecommunications, Blv. Carol I, nr, 11, Iasi, cod 700506, Romania; E-mail: ivpletea@etc.tuiasi.ro are DC capacitors. The inductors $\mathrm{L}_{R}, \mathrm{~L}_{\mathrm{S}}$ and $\mathrm{L}_{\mathrm{T}}$ have the same value, denoted by $\mathrm{L}_{1}$, and they are connected on the AC side. $L_{1}$ and $C$ fulfil the condition $0,05 \leq L_{1} C \omega^{2} \leq 0,10$ in order for the phase currents $i_{R}, i_{S}, i_{T}$ to be practically sinusoidal ( $\omega$ denotes the mains angular frequency), [7-9].

Considering that the currents $\mathrm{i}_{\mathrm{R}}, \mathrm{i}_{\mathrm{S}}, \mathrm{i}_{\mathrm{T}}$ are practically sinusoidal and have the amplitude $\mathrm{I}_{(1)}$, a function of the load 


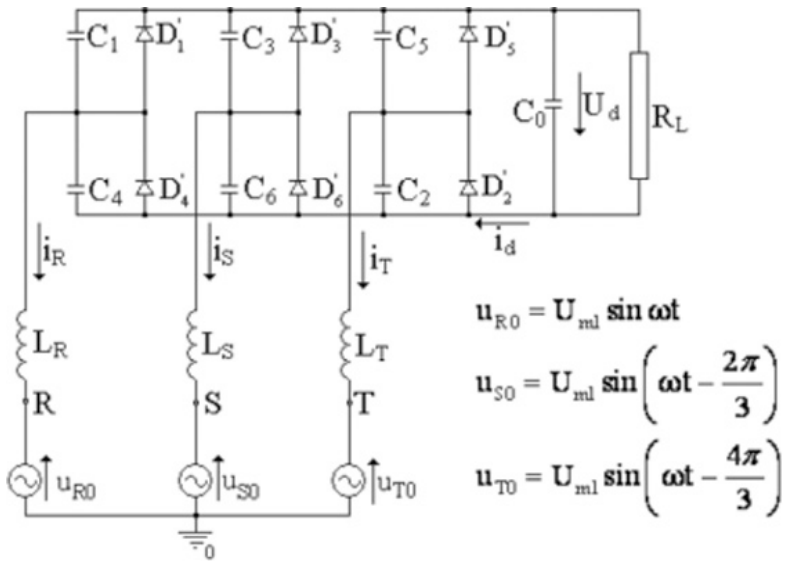

Fig. (2). Configuration of RNSIC converter.

resistor $R_{L}$, the current $I_{d}$ of medium value can be calculated from the following relation:

$I_{d}=\frac{3 I_{(1)}}{2 \pi}\left(1+\cos \omega t_{1}\right)$

where $\omega \mathrm{t}_{1}$ is the angle of turning on for the diodes $D_{1}^{\prime}-D_{6}^{\prime}$.

There are two extreme case during RNSIC converter functioning. In the first case, if $R_{L}=0$ (and so, $U_{d}=0$ and $\left.\omega t_{1}=0\right)$, the capacitors $\mathrm{C}_{1}-\mathrm{C}_{6}$ are short - circuited and the angle $\varphi=+90^{\circ}$ is inductive. In this case, the phase currents are sinusoidal and have maximum amplitude, equal to $I_{\max }$. In the second case, if the voltage $U_{d}$ exceeds the value $\sqrt{3} U_{m 1} /\left(1-2 L_{1} C \omega^{2}\right)$, the diodes $D_{1}^{\prime}-D_{6}^{\prime}$ do not conduct any more and the angle $\varphi=-90^{\circ}$ is capacitive (and so $R_{L}=\infty$ and $\left.\omega t_{1}=\pi\right)$. For this latter case, the phase currents are also sinusoidal and the amplitude has a minimum value $I_{\min }$, referred to as the holding current. The ratio $I_{\min } / I_{\max }$ has the value:

$\frac{I_{\min }}{I_{\max }}=\frac{2 L_{1} C \omega^{2}}{1-2 L_{1} C \omega^{2}}$

The phase displacement angle between the phase voltage and the fundamental of the phase current, as a function of the mean rectified voltage $U_{d}$ rated to the reference value $U_{r e f}=\frac{3 \sqrt{3} U_{m 1}}{\pi}$ specific for the classical three - phase rectifier [1]. The voltage $U_{d}$ can be established at a certain value by the load current.

The variations of the output voltage $U_{d}$ rated to the reference value $U_{\text {ref }}$ and the amplitude of the phase current $I_{(1)}$ rated to the reference value $I_{\max }$ as a function of the ratio $R_{L} / R_{L r}\left(\mathrm{R}_{\mathrm{Lr}}\right.$ denotes the rated load resistor for $\left.\varphi=0^{0}\right)$.

The rated operation of the RNSIC converter is defined for $\varphi=0^{0}$ and $R_{L} / R_{L r}=1$. For this case, the variations of the rated angle $\left(\omega t_{1}\right)_{r}$, the angle corresponding to when diodes begin to conduct, and the ratio $R_{L r} / L_{1} \omega$, as function of the parameter $L_{1} C \omega^{2}$. The interval between $45^{\circ}$ and $60^{\circ}$ for $\left(\omega t_{1}\right)_{r}$ ensures a reduced content of higher harmonics for the input currents.

\section{DC/AC OPERATION MODE}

In what follows we describe the operation of the converter in Fig. (1a) as an inverter. During the first stage, which starts at $\mathrm{t}_{0}$, the transistor $\mathrm{T}_{1}$ begins to conduct and the capacitor $\mathrm{C}_{1}$, charged at initial voltage $\mathrm{U}_{\mathrm{in}}$, is discharged to final voltage, $\mathrm{U}_{\text {end }}$, while capacitor $\mathrm{C}_{4}$, initially charged at voltage $\left(U_{d}-U_{i n}\right)$, is charged to $\left(U_{d}-U_{\text {end }}\right)$ by the help of the oscillatory processes in which transistor $\mathrm{T}_{1}$ and inductor $\mathrm{L}_{2}$ take part. After the blocking of transistor $\mathrm{T}_{1}$, made at $\mathrm{t}_{1}$, the second stage begins, when the energy accumulated in inductor $\mathrm{L}_{2}$ is rapidly transferred to $\mathrm{DC}$ and $\mathrm{AC}$ sources through diode $\mathrm{D}_{4}$. Finally, in the third stage, which lasts between $t_{2}$ and $t_{3}$, the current $i_{U}$ is zero, and the current $i_{R}$ has a practically sinusoidal waveform, flowing through capacitors $\mathrm{C}_{1}$ and $\mathrm{C}_{4}$. At the end of this stage, capacitor $\mathrm{C}_{4}$ is charged at voltage $\mathrm{U}_{\mathrm{in}}$, and $\mathrm{C}_{1}$ at voltage $\left(U_{d}-U_{\text {in }}\right)$. Inductors $\mathrm{L}_{2}$ have values two times smaller than $\mathrm{L}_{1}$. For the case of operation in inverter mode, the voltage $\mathrm{U}_{\mathrm{d}}$ is considered to be $15-25 \%$ greater than for the case of rectifier system operation. Diodes $D_{1}^{\prime}-D_{6}^{\prime}$ are chosen according to the RNSIC component design specification, while the diodes $D_{1}-D_{6}$ are rated for much smaller average currents.

The current $i_{R}$ is given by :

$i_{R}=\frac{u_{U 0}-u_{R 0}}{j \omega L_{1}}$

while its active value, $\mathrm{i}_{\mathrm{Ra}}$, is given by :

$i_{R a}=\frac{U_{m 2}}{\omega L_{1}}\left[\sin (\omega t+\alpha)-\frac{U_{m 1}}{U_{m 2}} \sin \omega t\right]$

The active power transferred to the AC source is given by:

$P=\frac{3}{2 \pi} \int_{0}^{2 \pi} i_{R a} U_{m 1} \cos \omega t d \omega t=\frac{3 U_{m 1} U_{m 2}}{2 \omega L_{1}} \sin \alpha$

In order to obtain a unitary power factor at the $\mathrm{AC}$ source, it results from formula (4) that:

$\cos \alpha=\frac{U_{m 1}}{U_{m 2}}$

It results that the value of the power transmitted to the AC source could be varied by modifying the amplitude $\mathrm{U}_{\mathrm{m} 2}$ (thus the angle $\theta$ ) and the angle $\alpha$ (thus the angle $\beta$ ).

The switch of the converter in Fig. (1a) from the inverter operation mode to the rectifier operation mode and reverse can be rapidly accomplished during a utility grid cycle $T=\frac{2 \pi}{\omega}$ 


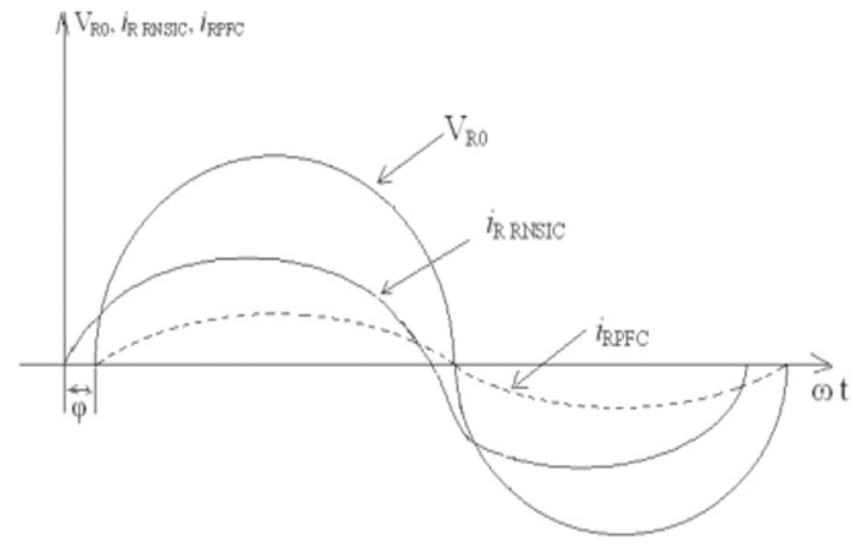

Fig. (3). Rectifier operation mode of the proposed converter (a) Waveforms of the phase current $i_{\text {RRNSIC }}$ and the phase voltage $v_{R 0}$ in steady - state; (b) Waveforms of the phase current $i_{\text {RPFC }}$

\section{REFERENCES}

[1] N. Mohan, T. Undeland., W. Robbins, "Power Electronics - Converters, Applications and Design", John Wiley \& Sons Inc., 1995, $200-248$.
[2] M. Kazmierkowski, R. Krishnan and F. Blaabjerg, "Control in power electronics selected problems", Academic Press, 2002, 89 208.

[3] W. Leonhard, Control of Electrical Drives, $2^{\text {nd }}$, edn Springer Verlag, Berlin, 1996

[4] H. Akagi, "Trends in Active Power Conditioners", IEEE Transactions on Power Electronics, 1994, 9, 263-268.

[5] D. Paice, "Power Electronics Converter Harmonics", IEEE Press, 1996, ISBN 07803-1137-X.

[6] D. Chung, and S. Sul, "Minimum-Loss Strategy for Three-Phase PWM Rectifier", IEEE Transactions on Industrial Electronics, 1999, 46, 517-526

[6] D. Alexa "Three-phase rectifier with almost sinusoidal input current", Electronics Letters, 2001, 37, 1148 - 1150.

[7] D. Alexa, A. Sirbu, and D. Dobrea,: "An analysis of three-phase rectifiers with near sinusoidal input currents", IEEE Trans. on Industrial Electronics, 2004, 51, 884-891.

[8] D. Alexa, A. Sirbu, D. Dobrea and T. Goras, "Topologies of threephase rectifiers with near sinusoidal input currents", IEE Proc. Electr. Power Appl., 2004, 151, 673-678.

Received: November 14, 2007

Revised: December 11, 2007

Accepted: December 18, 2007

(C) Pletea et al.; Licensee Bentham Open.

This is an open access article distributed under the terms of the Creative Commons Attribution License (http://creativecommons.org/license/by/2.5/), which permits unrestrictive use, distribution, and reproduction in any medium, provided the original work is properly cited. 